\title{
Gain vs phase in BOTDA setups
}

\author{
Alexia Lopez-Gil ${ }^{* a}$, Xabier Angulo-Vinuesa ${ }^{a}$, Marcelo A. Soto ${ }^{b}$, Alejandro Dominguez-Lopez ${ }^{\mathrm{a}}$, \\ Sonia Martin-Lopez ${ }^{\mathrm{a}}$, Luc Thévenaz ${ }^{\mathrm{b}}$, Miguel Gonzalez-Herraez ${ }^{\mathrm{a}}$ \\ a'Departamento de Electrónica, Universidad de Alcalá, Escuela Politécnica Superior, Campus \\ Universitario s/n, 28805 Alcalá de Henares, Spain; \\ ${ }^{b}$ EPFL Swiss Federal Institute of Technology, Institute of Electrical Engineering, SCI STI LT, \\ Station 11, CH-1015 Lausanne, Switzerland
}

\begin{abstract}
We evaluate the Brillouin frequency shift (BFS) determination error when using the Brillouin phase spectrum (BPS) instead of the Brillouin gain spectrum (BGS) in BOTDA setups. We compare the error obtained in the BFS determination in both cases, both with theoretical arguments and experimental data. In comparison to the gain, for an equal SNR and linewidth, the phase generally provides a better fit of the BFS for smaller frequency spans. This result opens a possible way to reduce the measurement time of certain BOTDA systems by using the phase feature.
\end{abstract}

Keywords: Fiber optics, Fiber optics sensors, Stimulated Brillouin scattering, Nonlinear optics.

\section{INTRODUCTION}

Conventional BOTDA configurations [1] exploit typically the gain/loss of the interaction, but rarely the induced phase shift. Strain or temperature variations in BOTDA systems are typically measured by fitting a quadratic curve to the gain/loss spectrum locally measured at each fiber position [2]. However the BPS is also a convenient way to determine the BFS, as it exhibits a sharp slope close to the BFS position [3]. Several techniques have been recently proposed to retrieve the longitudinal BFS profile by fitting a linear curve to the central spectral region of the BPS measured along the fiber [37], instead of using the classical BGS response measured by conventional BOTDA sensors. Nevertheless, it is unclear the conditions under which the linear fitting of the BPS provides better (or worse) accuracy in the BFS determination when compared to the use of the classical quadratic fitting over the BGS. In the literature, the performance of standard BOTDA sensors has been thoroughly analyzed and evaluated in terms of the best achievable error in the BFS determination [2]. However a similar analysis has never been provided for the phase profile case.

In this paper we present a comparison of the error produced when determining the BFS profile by employing BPS and BGS measurements. Theoretical and experimental results are compared in both cases. The results prove that the use of a linear fitting of the BPS can result in a better accuracy under specific conditions, especially when a small frequency range is used.

\section{ERROR IN THE BFS DETERMINATION BASED ON BPS MEASUREMENTS}

In a standard BOTDA system, the determination of the associated temperature/strain variations is performed estimating the center frequency position of the measured Lorentzian gain curve $\left(v_{B}\right)$ by fitting the BGS to a parabolic polynomial curve, as represented in Fig. 1a. Likewise, it is also possible to determine the BFS obtaining the frequency position at which the probe wave suffers no de-phase (see Fig. 1a) by fitting the BPS to a first-order polynomial curve.

An error in the frequency estimation of either the peak of the BGS or the zero de-phase of the BPS is typically produced due to the presence of noise in the measurements. In this work, a mathematical expression is obtained for the BFS determination error based on BPS measurements by following an approach based on the error propagation of a least-square linear fit [8] of the Brillouin phase response curve. Considering measurements with a certain SNR, a number of measured frequencies $\mathrm{N}$ (with a fixed step $\delta$ ) and a normalized gain response with a FWHM of $\Delta v_{B}$, the obtained expression for the error in the BFS determination is:

\footnotetext{
*alexia.lopez@uah.es; phone (+34)-91-885-69-14
}

Sixth European Workshop on Optical Fibre Sensors, edited by Elfed Lewis, Proc. of SPIE Vol. 9916, $991631 \cdot$ (C) 2016 SPIE · CCC code: 0277-786X/16/\$18 · doi: 10.1117/12.2236890 


$$
\sigma_{v-\text { linear }}(z)=\frac{1}{S N R} \cdot \frac{\Delta v_{B}}{p} \cdot \sqrt{\frac{1}{N}}
$$

Where $p$ is a form factor that depends on the shape of the input pulse spectrum, ranging typically from 1.5 to 2 . It should be noted that the second term in Eq. (1) has been obtained under the assumption that the curves have zero horizontal and vertical offset (i.e. the only error is introduced by noise). In general, if there exists either a vertical or horizontal offset on the phase curves, it will have a direct impact on the uncertainty in the BFS determination. Moreover, it can be shown that the impact of this offset term will be larger for smaller measurement spans. It is therefore important that BPS measurements are not biased by any offset, or that this offset is somehow corrected.

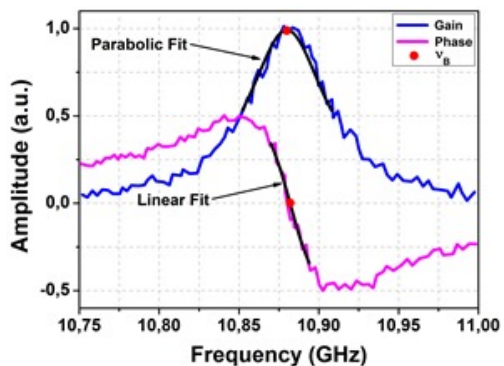

(a)

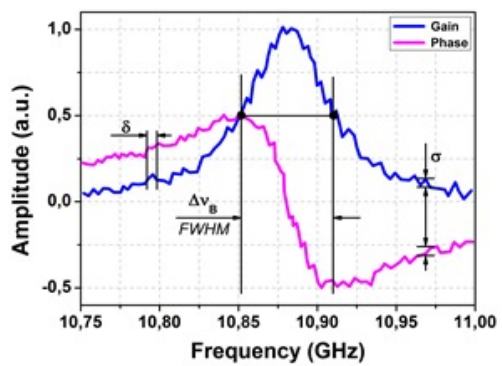

(b)

Figure 1. (a) Graphical representation of experimental gain and phase profiles obtained through a SI-BOTDA [7]. The position of the BFS is represented with a red dot as well as the necessary mathematical fits to determine it. (b) BFS determination error magnitude definition for the gain and phase profiles.

Unlike the estimated error using parabolic fitting [2], the expression in Eq. (1) does not depend on the frequency step used to scan the BPS. This can be explained by the fact that in the quadratic case the spectral range used to fit the BGS is essential to properly identify the curvature of the polynomial curve, having in this way a direct impact on the estimation of the BFS error. In the case of the a linear fitting the spectral width is not an explicitly important parameter, and what really defines the error in the BFS estimation, besides the SNR, is the number of points used in the fitting and the slope of the fitted line (which is related to the inverse of the FWHM of the BGS). Again, it should be clear that the assumptions used imply no vertical or horizontal offset. In case of such an offset, an inevitable error appears which does indeed depend on the measurement step. This error will be more important as the step is reduced.

\section{COMPARISON OF THE BFS ERROR IN THE GAIN AND PHASE CASES}

Equation (1) shows the dependence of the error obtained when determining the BFS ( $\left.\sigma_{v}\right)$ in the BPS case as a function of the number of points employed in the fitting $(N)$,the signal-to-noise ratio (SNR) and the Brillouin FWHM linewidth $\left(\Delta v_{B}\right)$. We will show a comparison of the evolution of both parabolic [2] and linear errors (Eq. (1)) as a function of these magnitudes. Experimental results are added in the comparison by introducing measurements using a SI-BOTDA [6]. This scheme provides a simple baseband method for determining simultaneously the gain and phase profiles of the SBS interaction along the fiber. Since BGS and BPS profiles are obtained from simple addition and subtraction of two independent measurements [7], the gain and phase responses are obtained with the same SNR, and the comparison is straightforward. Nevertheless, the conclusions of our study are absolutely general for any kind of phase-measuring BOTDA.

First, we analyze the dependence of both errors ( $\sigma_{v \text {-linear }}$ and $\sigma_{v \text {-parabolic }}$ ) on the number of measured frequency points $N$ around the BFS. The measurements were performed with $20 \mathrm{~ns}$ optical pump pulses, which led to a Brillouin linewidth ( $\Delta v_{B}$ ) of $56.7 \mathrm{MHz}$. The traces were averaged 300 times providing an equivalent SNR of 13.4. Fig. 5 shows a representation of each parabolic and linear error (experimental and theoretical for each case) for different frequency sampling steps ( $\delta=$ $0.25,1$, and $2 \mathrm{MHz}$ ). Obviously, as long as the sampling step increases the numbers of points to determine the error is consequently reduced (note that the number of points used in the fittings is in all cases bounded to a region of $\Delta v_{B}$ around the BFS). As can be observed, for an elevated number of sampling points $(N>100)$, and consequently a small sampling step ( $\delta=0.25 \mathrm{MHz}$ ), the error for both cases remains below $1 \mathrm{MHz}$ and fits quite well the theoretical tendency. As long 
as $N$ is reduced, the error rapidly increases for the parabolic case and the match among theory and experiment starts to diverge, in agreement with results reported in [2]. Contrary to this, in the linear case, the error remains quite bounded for almost all values of $N$ (note that for $N<10$ the results may not be statistically significant enough). It should be reminded that the linear fitting is only possible considering the scanned spectral points within the FWHM of the BGS (linear region of the BPS). For the linear case, as determined by Eq. 1, the error dependency with $\delta$ does not exist, what determines that even with a large sampling step $(3 \mathrm{MHz})$ and a low number of points $(\sim 10)$ the error is still close to $1 \mathrm{MHz}$. This is very interesting, as in any case this type of fitting could be used to reduce the number of sampling points and therefore also the measurement time.

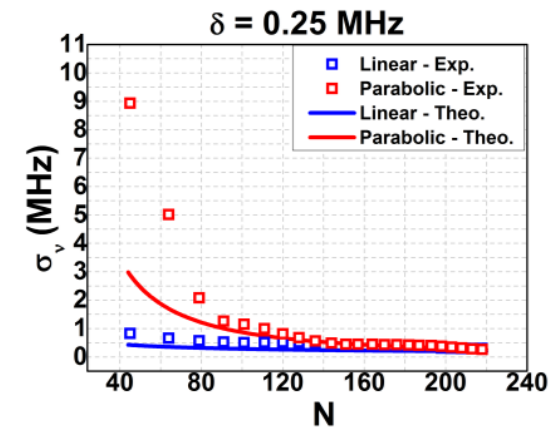

(a)

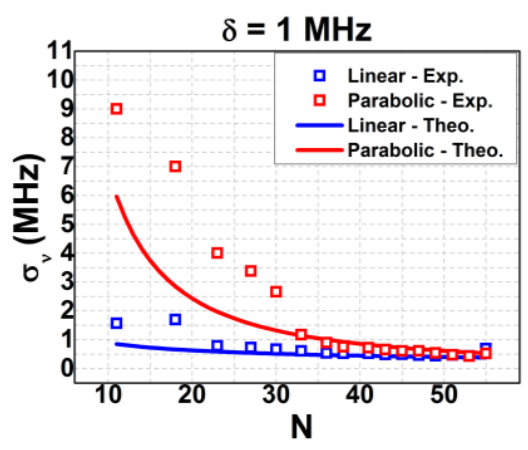

(b)

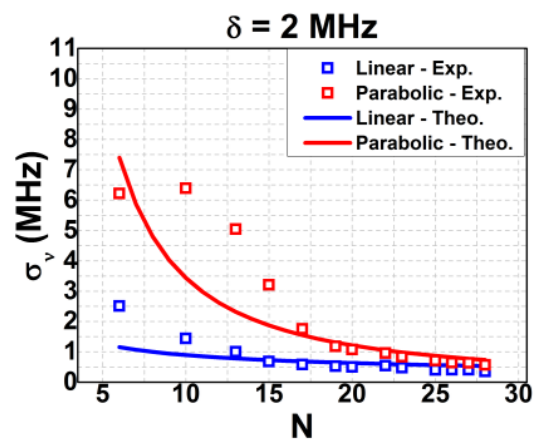

(c)

Figure 2. Linear and parabolic BFS determination error vs. the number of fitting points. The analysis has been performed for different frequency sampling steps ( $\delta=0.25,1$ and $2 \mathrm{MHz}$ ), with experimental results retrieved with $20 \mathrm{~ns}$ pulses and a 13.4 SNR equivalent to 300 average number. The results show theoretical simulations as well as experimental results.

Taking into account Eq. (1) and reference [2], if we represent the linear and parabolic errors as a function of the sampling step $\delta$, the linear error will in principle experience no variations as it is not affected by this variable. Fig. 3 shows the cited representation for the particular case of a spectral width of $56.7 \mathrm{MHz}(20$ ns optical pulses) and $N=18$ points. This particular number of points has been selected in order to be able to represent all the acquired sampling steps for the particular $\Delta v_{B}$ used. As it can be seen, the evolution of the error using the linear fit remains basically constant for frequency sampling steps greater than $1 \mathrm{MHz}$. For sampling steps smaller than $1 \mathrm{MHz}$, the offsets in the centering (vertical and horizontal) become non-negligible and have an increasing influence in the error as mentioned in the previous section. The parabolic fitting does show an inverse dependence with the frequency step in both the theoretical expectation and the experimental points. Again, the offsets have an increasing effect for smaller sampling steps, as it is visible in the divergence between theory and experiment as the sampling step is reduced.

It is interesting to compare mathematically when the use of linear or parabolic fitting should be preferable. If a ratio between both errors is performed, the following expression can be obtained:

$$
\frac{\sigma_{v-\text { parabolic }}(z)}{\sigma_{v-\text { linear }}(z)}=\frac{\sqrt{3} \cdot p}{2 \cdot \xi}=\frac{\sqrt{3} \cdot \Delta v_{B} \cdot p}{2 \cdot N \cdot \delta}
$$

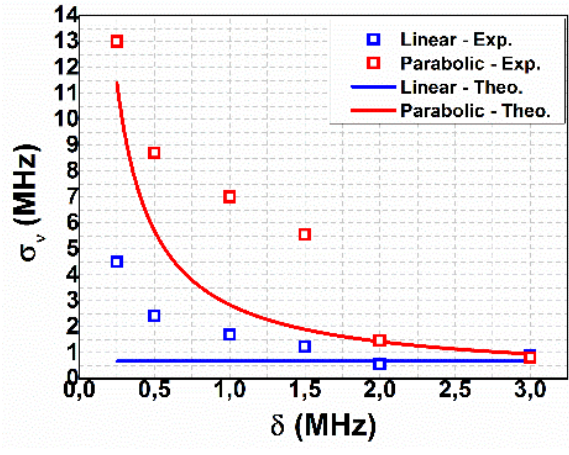

Figure 3. Error comparison as a function of $\delta$ for $20 \mathrm{~ns}$ pulses $\left(\Delta v_{B}=56.7 \mathrm{MHz}\right)$ and $N=18$. The linear error has no dependency with the sampling step, as predicted by equation 1 . Only experimentally diverges when $\delta<1 \mathrm{MHz}$.

As it can be seen, as the measured spectral span diminishes, linear fitting of the BPS becomes favored. When this quantity is larger than 1, it means that linear fitting of the BPS should be preferred. This condition is fulfilled when the normalized measurement $\operatorname{span}\left(\xi=N \cdot \delta / \Delta v_{B}\right)$, is smaller than $\sim 1.3$ (under our conditions). As a general tendency, considering that most of BOTDA applications need to perform a frequency sweep at least two times larger than $\Delta v_{B}$, with sampling steps 
close to $1 \mathrm{MHz}$, a large number of sampling points will normally be necessary, which benefits the parabolic fit. However, in some specific applications where small frequency sweeps could be feasible (homogeneous fibers, dynamic systems), the linear fit would be more beneficial.

\section{CONCLUSIONS}

In conclusion, we have evaluated theoretically and experimentally the performance a linear fitting of the BPS response against a parabolic fitting of the BGS in BOTDA setups as a function of the different experimental parameters (number of sampling points, SNR, sampling step and spectral width). To obtain the BFS from the BPS profile the simplest way is to perform a linear fit around the zero de-phase point of the SBS interaction. The results show that the linear fit used for the BPS profile performs better than the conventional parabolic one used for BGS measurements when the measured spectral span is small in comparison to the SBS width. However, these measuring conditions are not possible in all scenarios, as they would typically involve the need of a high-resolution setup and a homogeneous fiber with small BFS variations. Typically, for a robust sweep demanded in most applications, a measurement over twice the SBS width $\Delta v_{B}$ would be required, favoring the use of the parabolic fit over the BGS. One remarkable feature is that the error in the linear fit of the BPS has theoretically no dependency with the frequency-sampling step unlike the parabolic fitting of the BGS which does have a strong dependence on the sampling step.

It is clear that the BPS response is still under-explored for sensing applications. Considering the above conclusions, we believe that the BPS measurement may open the way to achieving a reduced measurement time in certain distributed Brillouin sensors, by simply reducing the number of sampling points required to achieve the same BFS determination error. This is clearly an important aspect to be explored in the future.

\section{ACKNOWLEDGMENTS}

This work was supported by the ERC through Starting Grant U-FINE (grant 307441), the Spanish "Plan Nacional de I+D $+\mathrm{i}$ " through projects TEC2012-37958-C02-01/02 and TEC2013-45265-R and the Comunidad de Madrid under projects EDISON (CCG2014/EXP-072) and SINFOTON-CM:S2013/MIT-2790. S. Martin- Lopez acknowledges support from the Spanish "Ministerio de Economía y Competitividad" through a "Ramón y Cajal” contract.

\section{REFERENCES}

1. Horiguchi, T. and Tateda, M., "BOTDA-nondestructive measurement of single-mode optical fiber attenuation characteristics using Brillouin interaction: theory," J. Lightwave Technol. 7(8), 1170-1176 (1989).

2. Soto, M. A., and Thévenaz, L., "Modeling and evaluating the performance of Brillouin distributed optical fiber sensors," Opt. Express 21(25), 31347-31366 (2013).

3. Urricelqui, J., Zornoza, A., Sagues, M., and Loayssa, A., "Dynamic BOTDA measurements based on Brillouin phaseshift and RF demodulation," Opt. Express 20(24), 26942-26949 (2012).

4. Dossou, M., Bacquet, D., and Szriftgiser, P., "Vector Brillouin optical time-domain analyzer for high-order acoustic modes," Opt. Letters 35(22), 3850-3852 (2010).

5. Lu, X., Soto, M. A., Gonzalez-Herraez, M., and Thévenaz, L., "Brillouin distributed fibre sensing using phase modulated probe,” Proc. SPIE 8794, 87943P-1 - 87943P-4 (2013).

6. Lopez-Gil, A., Angulo-Vinuesa, X., Dominguez-Lopez, A., Martin-Lopez, S., and Gonzalez-Herraez, M., "Exploiting nonreciprocity in BOTDA systems," Opt. Letters 40(10), 2193-2196 (2015).

7. Angulo-Vinuesa, X., Lopez-Gil, A., Dominguez-López, A., Cruz, J. L., Andres, M. V., Martin-Lopez, S., and Gonzalez-Herraez, M., "Simultaneous gain and phase profile determination on an interferometric BOTDA," Proc. of SPIE 9634, 24th International Conference on Optical Fibre Sensors, 963419 (2015).

8. Richter, P. H., "Estimating errors in least-squares fitting," Telecommun. Data Acquisition Prog. Rep. 42(122), 107137 (1995). 